TRABAJOS ORIGINALES

Rev Obstet Ginecol Venez. 2022; 82 (1): 67-72.

https://doi.org/10.51288/00820109

\title{
Posoperatorio de labioplastia de reducción de labios menores mediante técnica de incisión simple con láser $\mathrm{CO}_{2}$
}

\author{
Maryory Gómez, ${ }^{1}$ (iD) Oriana Hernández. ${ }^{1}$
}

\section{RESUMEN}

\begin{abstract}
Objetivo: Determinar los resultados posoperatorios de la reducción de labios menores mediante la técnica de incisión simple con láser $\mathrm{CO}$, en el periodo agosto 2015 - abril 2016, en la ciudad de Valencia, edo. Carabobo.

Métodos: Estudio descriptivo, no experimental y de campo. La muestra estuvo constituida por 50 pacientes que acudieron a la consulta de ginecología. Se utilizó para la recolección de los datos la técnica de observación directa, llenado de la historia clínica, y evaluación a los 7 días de posoperatorio.

Resultados: Una vez analizados los datos se obtuvo como resultados que la edad promedio de las pacientes fue 32,68 años. Entre los sintomas posoperatorios, el $8 \%$ refirió ardor en el área operatoria las primeras 24 horas. Las complicaciones posoperatorias fueron las siguientes: hematomas $4 \%$ y dehiscencia $2 \%$. El $98 \%$ de las pacientes se sintieron satisfechas o muy satisfechas del proceso.

Conclusión: Las complicaciones son menores con la técnica de incisión simple con láser CO2, la misma es segura y se obtuvieron resultados satisfactorios. Permite, además, resecar la zona hipercrómica del borde de los labios que, en la mayoría de los casos, suele incomodar desde el punto de vista estético. Las bondades de la herramienta incluyen una mejor cicatrización, menos sintomas posoperatorios y una recuperación más rápida.
\end{abstract}

Palabras clave: Labioplastia, Laser CO2, Técnicas de reducción de labios, Posoperatorio de labioplastia

\section{Post-operative reduction labioplasty of the labia minor using a simple incision technique with $\mathrm{CO}_{2}$ laser}

SUMMARY

Objective: To determine the postoperative results of the reduction of labia minora using the simple incision technique with $\mathrm{CO}_{2}$ laser, in the period August 2015 - April 2016, in the city of Valencia, Carabobo State.

Methods: Descriptive, non-experimental and field study. The sample consisted of 50 patients who attended the gynecology consultation. The technique of direct observation, filling of the clinical history, and evaluation at 7 days postoperatively was used for data collection.

Results: Once the data were analyzed, it was obtained as results that the average age of the patients was 32.68 years. Among the postoperative symptoms, $8 \%$ reported burning in the operative area in the first 24 hours. Postoperative complications were as follows: bruising 4\% and dehiscence 2\%. $98 \%$ of patients felt satisfied or very satisfied with the process.

Conclusion: Complications are minor with the simple incision technique with $\mathrm{CO}_{2}$ laser, it is safe and satisfactory results were obtained. It also allows to dry out the hyperchromic area of the edge of the lips that, in most cases, tends to bother from the aesthetic point of view. The benefits of the tool include better healing, fewer postoperative symptoms and faster recovery.

Keywords: Labioplasty, Laser $\mathrm{CO}_{2}$, Reduction technique labia minora, Postoperative labioplasty.

${ }^{1}$ Especialistas en Obstetricia y Ginecología. Centro Clínico Guerra Méndez. Valencia. Venezuela. Correo para correspondencia: dragomez@ginecoestetica.com Forma de citar este artículo: Gómez M, Hernández O. Posoperatorio de labioplastia de reducción de labios menores mediante técnica de incisión simple con láser CO2. Rev Obstet Ginecol Venez. 2022; 82(1): 67-72. https://doi.org/10.51288/00820109

\section{INTRODUCCIÓN}

Hoy en día la ginecología estética, ha despertado gran interés, tanto en el gremio de especialistas en Ginecología y Obstetricia, como en las mujeres. Dentro de los procedimientos más solicitados se ubica la labioplastia de reducción, el cual se indica 
en aquellas pacientes que tengan labios hipertróficos, labios menores que pueden medir más de $4 \mathrm{~cm}$ y presentar asimetría de bordes, como consecuencia de condiciones congénitas, irritación crónica o estímulo androgénico excesivo, aunque en algunos casos la demanda es por causas funcionales (irritación, molestia mecánicas con la deambulación o las relaciones sexuales) (1).

La presencia de uno o más síntomas constituyen elementos para indicar la labioplastia, pero esta también, puede hacerse con fines estéticos, de acuerdo con Arredondo y cols. (2), cuando se presentan labios menores expuestos, que aunque no afecten el desarrollo de la vida íntima y cotidiana de la mujer, si menoscaban su autoestima, tratándose por tanto de una intervención, cuya única misión es mejorar el aspecto de la vulva y la relación de la paciente con su cuerpo.

En la actualidad distintas técnicas han sido implementadas y se diferencian entre sí por el tipo de incisión en el labio. Dentro de ellas, González (3) describe la Z-plastia, la técnica de Wedge, también llamada en "V", la técnica de Trim o incisión simple, la técnica de Alter, y la técnica de Choi. En tal sentido, la técnica lineal de Trim, es una de las más utilizadas por los cirujanos desde 1999, con una curva de aprendizaje mucho más corta que las demás técnicas, la cual consiste en realizar un corte longitudinal de labio, en función a lo que indica Ellsworth y cols. (4), con la extracción de la porción hipercrómica redundante y la aproximación de la herida mediante sutura absorbible. Existen estudios que detallan los desafortunados resultados, poco naturales, la pérdida de la sensibilidad, dolor e incomodidad que esta técnica pudiera ocasionar (5). De allí que hayan surgido otras técnicas para la realización de la reducción de labios menores.

Es importante destacar que hay diferentes puntos de vista, con respecto a la utilización de la técnica simple y debido a la experiencia que las autoras tienen en la consulta, entre agosto 2015 a abril 2016, se logró realizar la reducción de labios menores por la técnica de incisión simple, con láser $\mathrm{CO}_{2}$, a un grupo etario entre 14 y 56 años, cuyos resultados posoperatorios se presentan.

Como basamento teórico para darle valor a la investigación se pudo evidenciar, que Oranges y cols. (6) realizaron una revisión exhaustiva de la literatura de 38 estudios publicados entre 1971 y 2014, con una representación de 1981 pacientes, en donde se estimaron las diferentes técnicas aplicadas en la reducción de labios menores, obteniendo que en las 8 técnicas aplicadas, se presentaron pocas complicaciones posoperatorias y en un pequeño número de casos requirieron revisión quirúrgica o resección secundaria.

Muchos estudios de estas técnicas para reducir los labios menores se han publicado en las últimas décadas, incluyendo informes de casos y series de casos retrospectivos, como los de Barba (7), pero hasta mayo 2015, no ha habido ningún estudio de las complicaciones generales o la tasas de satisfacción asociadas con el amplio espectro de técnicas. Sin embargo, en vista de las controversias con los diferentes tipos de técnicas y las pocas publicaciones de consecuencias en el posoperatorio, se decidió investigar sobre la ventaja de trabajar con láser y las posibles complicaciones o síntomas que puedan manifestar las pacientes, luego de la labioplastia de reducción de labios menores con técnica de incisión simple con láser $\mathrm{CO}_{2}$. Es por ello que la investigación se hizo para determinar los resultados posoperatorios de la labioplastia de reducción de labios menores, mediante la técnica de incisión simple con láser $\mathrm{CO}_{2}$, identificar las complicaciones, revelar los síntomas que presentan las pacientes en el posoperatorio y describir la satisfacción de las mujeres que se someten a este tipo de cirugías. 


\section{POSOPERATORIO DE LABIOPLASTIA DE REDUCCIÓN DE LABIOS MENORES \\ MEDIANTE TÉCNICA DE INCISIÓN SIMPLE CON LÁSER CO2}

\section{MÉTODOS}

Se realizó un estudio descriptivo, el diseño adoptado es el no experimental, de campo (8). La población estuvo constituida por pacientes que acudieron a la consulta de ginecología del Centro Médico Guerra Méndez, en el periodo agosto de 2015 y abril de 2016, las cuales manifestaron problemas funcionales $\mathrm{y}$ estéticos de su zona intima. La muestra seleccionada fue de tipo no probabilística, de voluntarias, y estuvo constituida por 50 pacientes que fueron sometidas a labioplastia de reducción mediante técnica de incisión simple con láser $\mathrm{CO}_{2}$.

Para la recolección de datos se utilizó la observación directa (9), en un primer momento se llenó la historia clínica a cada una de las pacientes previa a la intervención quirúrgica. Posteriormente, se llevó a efecto una evaluación clínica a los 7 días de posoperatorio, determinando los síntomas o molestias, así como las posibles complicaciones, y transcurridos 120 días de haberse realizado la cirugía, se efectuó otra evaluación. Se utilizó una escala de satisfacción, en la que la paciente expresaba con una puntuación del 1 al 4 como se autocalificaba, donde el número 1 era igual a no satisfecha, 2 a poco satisfecha, 3 es satisfecha y 4 muy satisfecha, de acuerdo con Crépaux y cols. (10).
Una vez recolectados los datos se sistematizó la base de datos Microsoff Excel, donde se analizaron los datos con las técnicas de estadísticas descriptivas mediante tablas y gráficos.

\section{RESULTADOS}

Se registró una edad promedio de 32,68 $\pm 10,06$ años, con un registro mínimo de 14 y un máximo de 56 años.

En el gráfico 1 se puede observar que en 47 casos (94\%) no se presentaron complicaciones posoperatorias; dos pacientes $(4 \%)$ presentaron hematomas y $1(2 \%)$ presentó dehiscencia (1 caso).

Con relación a los síntomas posoperatorios, 46 pacientes (92\%) estaban asintomáticas y 4 (8\%) indicaron ardor en el área operatoria las primeras 24 horas (gráfico 2).

La evaluación de la satisfacción se presenta en el gráfico 3. Se observa que en 49 casos $(98 \%)$ las pacientes estaban satisfechas: 33 (66\%) muy satisfechas y 16 (32\%) satisfechas. Solo 1 paciente $(2 \%)$ dijo sentirse poco satisfecha.

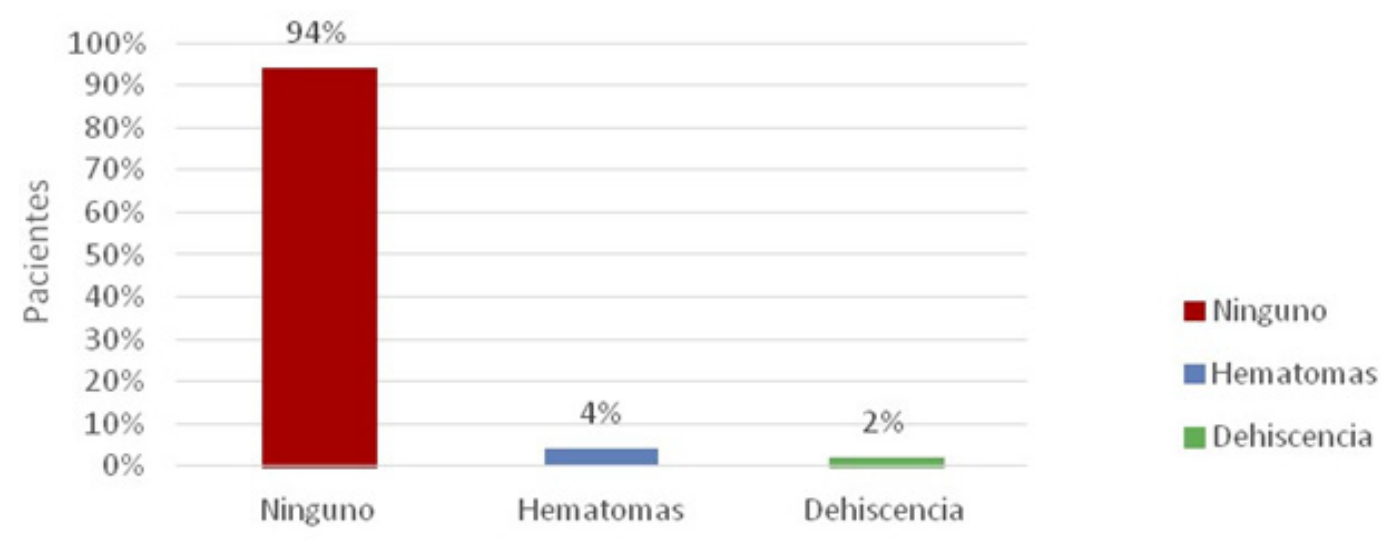

Gráfico 1. Distribución porcentual de pacientes según las complicaciones presentadas 
- Ninguno = ardor en el área Operatoria

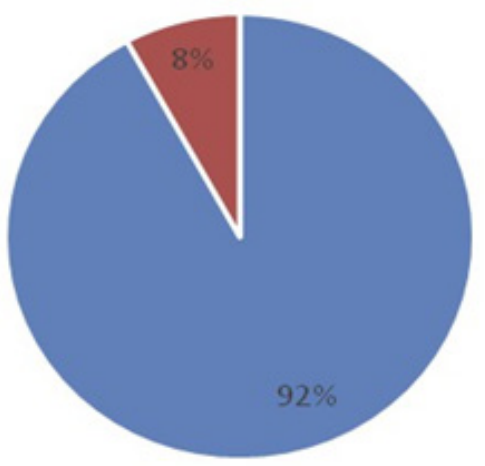

Gráfico 2. Distribución porcentual de pacientes según los síntomas presentados en el posoperatorio

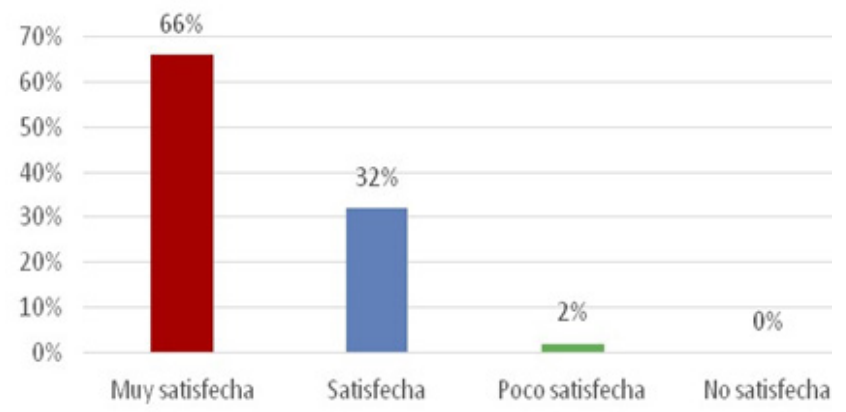

Gráfico 3

Distribución porcentual de pacientes según la satisfacción experimentada con la técnica

\section{DISCUSIÓN}

Estos resultados evidencian que la técnica de incisión simple con láser $\mathrm{CO}_{2}$, es una práctica quirúrgica segura donde las complicaciones se pueden disminuir mediante ella. Además, es de gran importancia la utilización como recurso del láser $\mathrm{CO}_{2}$, ya que en la mayoría de los estudios sobre labioplastia de reducción de labios menores, no describen con exactitud la técnica utilizada para realizar la misma. En aporte a este estudio, Cayrac y Rouzier (11), en 2012, realizaron la evaluación de la reducción de labios menores por resección longitudinal, no obteniendo ninguna consecuencia negativa en 26 casos estudiados, en los que la mayoría estaban satisfechas con la cirugía, describiendo solo una paciente no satisfecha por presentar pérdida de la flexibilidad del clítoris. Asimismo, en el estudio realizado por el Pardo y cols. (1) entre octubre 2003 y abril 2014, que incluyó 500 casos intervenidos bajo la misma técnica, utilizando láser diodo en la mitad y electrobisturí en la otra mitad, reportaron solo dos casos de sangrado moderado posoperatorio, no controlable y en 44 casos se produjo dehiscencia de la herida.

Los resultados demuestran que la técnica de incisión simple con láser $\mathrm{CO}_{2}$ permite una recuperación no traumática y segura para las pacientes que se someten a este tipo de cirugías, lo que las lleva a menor tiempo de inactividad, permitiendo que las mismas se incorporen a sus actividades diarias luego de las 72 horas de haber sido operadas. En este orden de ideas, Pardo y cols. (1) reportaron que solo 14 de las 500 pacientes evaluadas refirieron dolor moderado por más de una semana, el cual se resolvió espontáneamente, sin embargo, registraron un caso de dolor prolongado el cual correspondió a una paciente con labios menores gruesos, en quien se hizo resección del tejido conectivo-celular con el objetivo de adelgazarlos. En esta serie, solo $8 \%$ de las pacientes refirieron ardor en el área operatoria, y en todos los casos, se resolvió en las primeras 24 horas.

La técnica de incisión simple con láser $\mathrm{CO}_{2}$, es una alternativa que ofrece un buen proceder. Las pacientes que se mostraron muy satisfechas habían expresado que su motivo de consulta fue más por molestia funcional que estética. La paciente que respondió, "poco satisfecha" lo relacionó con el resultado estético que pudo corregirse en una segunda consulta, de acuerdo a lo expresado por González-Isaza y cols. (12). 


\section{POSOPERATORIO DE LABIOPLASTIA DE REDUCCIÓN DE LABIOS MENORES \\ MEDIANTE TÉCNICA DE INCISIÓN SIMPLE CON LÁSER CO2}

Al considerar los resultados de esta investigación se relacionan con estudios como el Oranges y cols. (6), quienes realizaron una revisión exhaustiva de las diferentes técnicas que se utilizaban en la labioplastia de reducción, en publicaciones durante los años 1971 al 2014, allí se pudo determinar que los resultados son satisfactorios a juzgar a partir del examen clínico, puesto que al valorarse las 8 técnicas, mostraron pocas complicaciones posoperatorias, aunque, en un pequeño número de casos, las complicaciones requirieron revisión quirúrgica o resección secundaria.

Lallemant y cols. (13), en 2019, realizaron la reducción de labios menores en 92 pacientes, entre 2010 y enero de 2017. Lamentablemente, solo 37 (40 \%) dieron respuesta a una encuesta aplicada posterior a la cirugía, entre ellas, se encontró un $86 \%$ de pacientes satisfechas con los resultados.

En el presente grupo de pacientes, las 49 que expresaron sentirse satisfechas, habían consultado por síntomas funcionales, como dolor y molestia durante la penetración, realizar ejercicios, el roce al caminar, inflamación de los labios al utilizar toallas sanitarias durante la menstruación. La intervención resultó en un impacto positivo en estas pacientes, mejorando su calidad de vida.

Una ventaja que se observó en las pacientes intervenidas, es que se pudo resecar la zona hipercrómica del borde de los labios, que en la mayoría de los casos suele incomodar desde el punto de vista estético. Se agregan las bondades de la herramienta, como en este caso el láser $\mathrm{CO}_{2}$, que permite tener una mejor cicatrización, menos síntomas posoperatorios y una recuperación más rápida, llevando a una incorporación precoz a sus actividades sin implicaciones.

La presente investigación representó una experiencia de gran significado, desde la motivación y porque desde hace 8 años aproximadamente se comenzó a observar un aumento de los estudios con respecto a la práctica de la ginecología estética, en especial en la cirugía más común como lo es la labioplastia de reducción de labios menores, describiéndose diferentes técnicas y resultados posoperatorios, en los que algunos autores reportaban mayor frecuencia de complicaciones y resultados poco estéticos con la técnica de incisión simple o de Trim.

\section{CONCLUSIONES}

La técnica simple, utilizando el láser $\mathrm{CO}_{2}$, es segura, bien tolerada, produce pocos síntomas posoperatorios, se asocia a pocas complicaciones, todas leves, y produce satisfacción en las pacientes en quienes se emplea.

Para futuros estudios, se recomienda realizar comparación entre técnicas y considerarse como prioridad la herramienta utilizada como el láser, electrobisturí o bisturí.

\section{Sin conflictos de interés.}

\section{REFERENCIAS}

1. Pardo J, Solá V, Galán G, Contreras L. Labioplastia genital experiencia y resultados en 500 casos consecutivos. Rev Chil Obstet Ginecol. 2015; 80(5). doi.org/10.4067/S0717-75262015000500007

2. Arredondo R, Mateos J, Zuñiga D, Alatriste D. Procedimientos cosméticos en ginecología. Acta Médica Grupo Ángeles [Internet]. 2012 [febrero de 2017]; 10 (2): 80-86. Disponible en: https://www. medigraphic.com/pdfs/actmed/am-2012/am122c.pdf.

3. González PI. Classification of hypertrophy of labia minora: Consideration of a multiple component approach. Surg Technol Int [Internet] 2015 [febrero de 2017]; 27:191-194. Disponible en: https:// surgicaltechnology.com/27-Gynecology.htm\#662

4. Ellsworth WA, Rizvi M, Lypka M, Gaon M, Smith B, Cohen B, et al. Techniques for labia minora reduction: an algorithmic approach. Aesthetic Plast Surg. 2010; 34(1):105-110. doi: 10.1007/s00266-009-9454-5. 
5. Casale C, Casale D, Tébar A, Sánchez JJ, González B, Herrera A. Reducción funcional y estética de los labios menores: labioplastia con técnica de resección con colgajos especulares en «S». Clín Invest Gin Obst. 2014; 41(2):80-82. doi:10.1016/j.gine.2012.12.012

6. Oranges CM, Sisti A, Sisti G. Labia minora reduction techniques: a comprehensive literature review. Aesthet Surg J. 2015; 35(4):419-431. doi: 10.1093/asj/sjv023.

7. Barba MJ. Labioplastia con láser, lo último en cirugía íntima [Internet]. Madrid: Clínica Médica Estética Doctora Barba; [febrero de 2017]. Disponible: https:// www.doctorabarba.com/cirugia-intima/labioplastiamadrid.html

8. Londoño J. Metodología de la investigación epidemiológica. 3a. ed. Bogotá: Editorial Manual Moderno S.A; 2004.

9. Arias F. El proyecto de investigación. 4a. ed. Caracas: Editorial Episteme; 2004.

10. Crépaux V, Legendre G. [Labiaplasty: Motivations and post-surgery satisfaction]. Gynecol Obstet Fertil Senol. 2019; 47(6):489-496. French. doi: 10.1016/j. gofs.2019.04.005.
11. Cayrac M, Rouzier R. [Evaluation of labia minora reduction by longitudinal resection]. Gynecol Obstet Fertil. 2012; 40(10):561-565. French. doi: 10.1016/j. gyobfe.2011.08.004.

12. González-Isaza P, Lotti T, França K, Sanchez-Borrego $\mathrm{R}$, Tórtola JE, Lotti J, et al. Carbon dioxide with a new pulse profile and shape: a perfect tool to perform labiaplasty for functional and cosmetic purpose. Open Access Maced J Med Sci. 2018; 6(1):25-27. doi: 10.3889/oamjms.2018.043.

13. Lallemant M, Porté C, Laroche J, Andreoletti JB, Gay C. [Functional or aesthetic labia minora reduction: Complications, revision surgeries and results - a comparative study]. Gynecol Obstet Fertil Senol. 2019; 47(4):330-336. French. doi: 10.1016/j. gofs.2019.02.004. 